\title{
A RARE CASE OF EMPHYSEMATOUS SPINAL OSTEOMYELITIS
}

\section{General Medicine}

Dr. Shilpa S. Kuthe Assistant Professor Department of General Medicine, NKP Salve Institute of Medical (Tulankar)* Sciences \& Lata Mangeshkar Hospital and Research Centre, Hingna, Nagpur, Maharashtra. ${ }^{*}$ Corresponding Author

Dr. Nalini R. Humaney

Professor and Head, Department of General Medicine, NKP Salve Institute of Medical Sciences \& Lata Mangeshkar Hospital and Research Centre, Hingna, Nagpur, Maharashtra.

Dr. Atharva Junior Resident, Department of General Medicine, NKP Salve Institute of Medical Chintawar Sciences \& Lata Mangeshkar Hospital and Research Centre, Hingna, Nagpur, Maharashtra.

\section{ABSTRACT}

Emphysematous Osteomyelitis of the vertebrae is an extremely rare clinical presentation and is associated with significant morbidity and mortality. Here we report a case of a 52 years old man who presented with acute transverse myelopathy and was already started on anti-tubercular therapy for suspected Pott's spine before being referred to us. We found him to have emphysematous osteomyelitis involving the thoracic vertebrae. We treated him with empirical antibiotics and surgical drainage, tissue culture revealed Escherichia coli. Early diagnosis and immediate surgical decompression are necessary for better outcomes in cases of Emphysematous Osteomyelitis.

\section{KEYWORDS}

\section{Emphysematous Osteomyelitis; Escherichia coli; Vertebrae}

Introduction: Emphysematous osteomyelitis is an unusual condition characterized by the presence of intraosseous gas. A prompt diagnosis is required to expedite management as it is a potentially fatal condition. Multiple comorbidities such as diabetes mellitus, malignancy, alcohol abuse, Crohn's disease and other conditions causing immunosuppressi on, predispose to this condition. Generally, anaerobes or members of the Enterobacteriaceae family are the causative organisms; however, the aetiology can be mono or polymicrobial.

Case Report: A 52 years old man, chronic alcohol consumer, was referred to us as a case of Pott's spine and already started on antitubercular therapy (ATT) and steroids for two weeks. A thorough history taking revealed that the patient had an acute onset of paraplegia two weeks ago after he returned home from his morning walk. The weakness was associated with bowel and bladder incontinence and a complete sensory loss below D10 dermatome. There was no history suggestive of root pains, backache or fever. On examination, he was icteric, hemodynamically stable, conscious and oriented. There was no gibbus or paraspinal tenderness. Superficial bedsore was present on lower back. Lower limbs were flaccid with areflexia and plantars were extensors bilaterally. On respiratory system examination, findings were suggestive of a right sided Pleural Effusion. On enquiry, he denied any history of cough/fever/weight loss/anorexia.

On investigations, there was normochromic normocytic anaemia, no leucocytosis, ESR was $21 \mathrm{~mm}$. His plasma glucose was normal (non diabetic), HIV non-reactive. Liver function tests revealed mild direct hyperbilirubinemia, transaminitis (ATT induced hepatitis) and hypoalbuminemia. MRI Dorsolumbar spine(outside) was suggestive of early osseous destruction at D 7,8,9 with involvement of intervening disc; also, involvement of L4,5 and soft tissue edema with small collection in epidural space -? Pott's spine, ??metastases. Contrast enhanced CT scan of thorax showed multiple air foci involving the T7, T8 andT9 vertebral bodies extending to involve pre and paravertebral spaces and into anterior epidural space causing cord compression at this level. Also moderate right sided pleural effusion with underlying collapse consolidation. Pleural fluid analysis showed exudative characteristics with high protein content $(3.7 \mathrm{~g} / \mathrm{dl})$ and high $\mathrm{LDH}$ (2179U/L). CSF analysis revealed viscous CSF on lumbar puncture showing 12 lymphocytes, hypoglycorrhachia, high proteins $(1613 \mathrm{mg}$ / dl) (? pseudo-Froin's syndrome) and ADA of 11.4U/L. Ultrasound abdomen shower liver parenchymal disease with ascites.

He was started on broad spectrum antibiotics: injectable Meropenem $1 \mathrm{~g}$ thrice daily, Clindamycin $600 \mathrm{mg}$ thrice daily and Metronidazole $400 \mathrm{mg}$ thrice daily. ATT was continued. Spinal Surgery was planned after explaining meagre chance of neurological recovery. During surgery, no foul smell was noted to suggest anaerobic infection, minimal epidural fluid found, organised yellowish membrane found in epidural space anterior to spinal cord -fluid, tissue and bone biopsy sent for analysis; spinal decompression with fixation was done. Tissue culture showed growth of Escherichia coli sensitive only to carbapenem. Bone biopsy showed necrotic tissue with suppurative inflammation, Ziehl-Neelsen stain for acid fast bacilli was negative. Neurological weakness did not improve after surgery, patient was given 4 weeks course of antibiotics (Meropenem) and discharged.
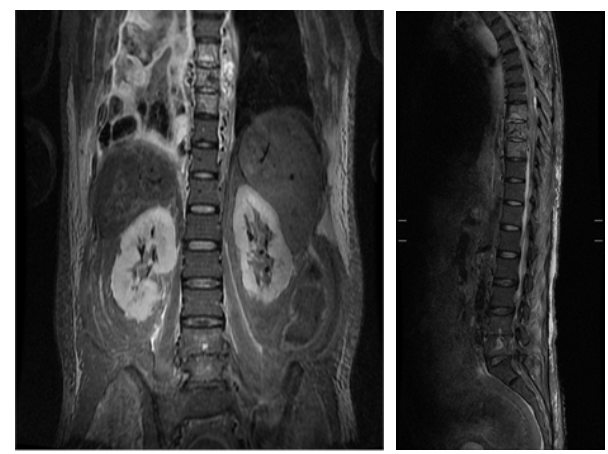

Figure 1:MRI Thoracolumbar spine showing osseous destruction at D7,8,9 and small epidural collection with cord compression.

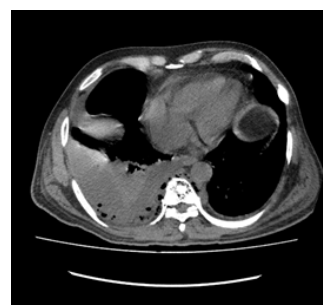

Figure 2: CECT Thorax showing multiple air foci involving $T 7,8,9$ vertebral bodies extending into epidural space. right sided moderate pleural effusion also seen.

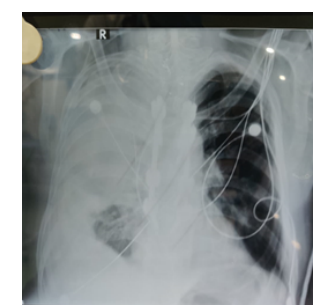

Figure 3 showing Xray chest $P A$ view after spinal rod and screw fixation.

\section{DISCUSSION:}

Intraosseous gas was initially described as a sign of osteomyelitis in $1981^{1}$. When seen in the extra-axial skeleton, intraosseous gas is 
virtually pathognomonic for emphysematous osteomyelitis; in rare cases the differential diagnosis includes trauma, post-surgical change, lymphangiomatosis of the bone, degenerative disease, osteonecrosis, and neoplasm. ${ }^{2}$ Conversely, intraosseous gas seen in vertebral bodies is almost always due to a non-infectious cause. Indeed, intravertebral gas was long considered to exclude infection, with almost all cases due to degenerative disease, or less commonly, osteonecrosis or a neoplastic process. ${ }^{3,4}$ Osteomyelitis is likely when extensive intravertebral gas, bone edema, and/or adjacent collections are present ${ }^{5}$. When emphysematous osteomyelitis is seen, empiric treatment should include antibiotic(s) with activity against anaerobes and members of the Enterobacteriaceae family. And surgical treatment should be considered in acute osteomyelitis if there is finding of abscess formation or radiologic evidence of necrosis, or if the patients do not respond to antimicrobial agents ${ }^{6}$. In most cases, the infection occurs by hematogenous spread, however may also be related to contagious spread from an intraabdominal source of infection, or from a skin or soft tissue source of infection; or following intra-abdominal or spinal surgery. ${ }^{7}$

In our patient, who was not immunocompromised, absence of fever, lack of pain or spinal tenderness and an acute onset of symptoms were all misleading and inconclusive. Further, previous labelling of patient as Pott's spine caused some bias. Delay in treatment resulted in permanent neurodeficit.

Conclusion: A high index of suspicion for emphysematous osteomyelitis and early specific investigations and treatment can be lifesaving.

Conflict of interest: No conflict of interest to declare.

References:

1. Ram PC, Martinez S, Korobkin M, Breiman RS, Gallis HR, Harrelson JM. CT detection of intraosseous gas: a new sign of osteomyelitis. AJR Am J Roentgenol 1981; 137:721-3

2. Potocki J, Kaushik S, Mira JL. Anaerobic osteomyelitis of femoral head with intraosseous, intra-articular, bursal and muscle pneumotosis. Skeletal Radiol 2003; 32:46-8.

3. Resnick D, Niwayama G, Guerra Jr J, Vint V, Usselman J. Spinal vacuum phenomena: anatomical study and review. Radiology 1981; 139:341-8.

4. Bielecki DK, Sartoris D, Resnick D, Van Lom K, Fierer J, Haghigi P. Intraosseous and intradiscal gas in association with spinal infection: report of three cases. AJR Am J intradiscal gas in association

5. Luey C, Tooley D, Briggs S. Emphysematous osteomyelitis: a case report and review of the literature. Int J Infect Dis 2012;16:e216-20.

6. Korean Society for Chemotherapy, Korean Society of Infectious Diseases, Korean Orthopaedic Association. Clinical guidelines for the antimicrobial treatment of bone and joint infections in Korea. Infect Chemother 2014; 46:12538.

7. McDonnell O, Khaleel Z. Emphysematous osteomyelitis. JAMA Neurol 2014; 71:512. 